\section{CP'92 \\ International Conference on Computational Physics}

24-28 August 1992,

Prague, Czechoslovakia

CP '92 formed one in the series of conferences organized by the Computational Physics Interdivisional Group of the EPS and the Computational Physics Division of the American Physical Society, with the even years in Europe and the odd years in the USA. The first meeting took place in Boston in 1989; the second in Amsterdam in 1990; the third in San Jose last year, while next year's conference will be organized in Albuquerque, USA. Some 195 people from 24 different countries registered for CP ' 92 . Monday, the 24 th was devoted to tutorials on the processing of scientific documents, features of Fortran, neural networks, parallel algorithm design, cellular automata, visualization, symbolic computing, and networking a physics department. The Plenary sessions were held on the Tuesday. A fair part was in the form of two-minute introductions to posters, where it was remarkable how some speakers managed to interest the audience in their poster in such a short time. Wednesday and Thursday offered sessions and posters in parallel; there were only plenary sessions on the closing day.

Being a conference organized by an interdivisional group, the range of subjects was naturally quite broad. Session topics included (quantum) molecular dynamics, physics education, dynamical systems, electronic structure, fluids and plasmas, mesoscopic physics, Monte Carlo simulations of quantum systems, new trends in hardware and software, Monte Carlo techniques, self-organization and coherent structures, symbolic computing, nano computing, and non-equilibrium phase transitions and critical phenomena.

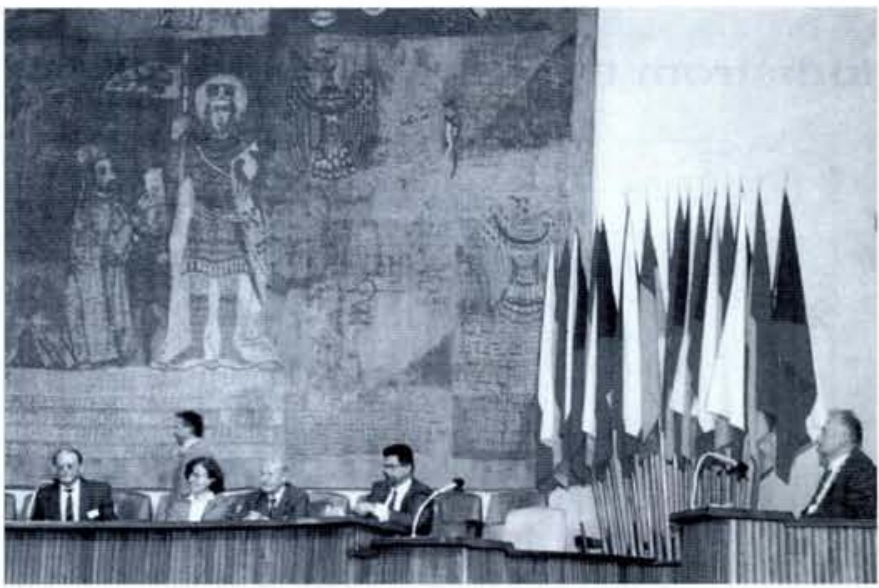

The CP'92 opening ceremony in the magnificient Aula Maxima of the University of Prague's Carolinum.

Some highlights of the conference are described by the authors themselves in accompanying boxes. The main trend of the conference was clearly dominated by the impact of massively parallel machines, both now, but especially in the rather near future, and how Europe can play a rôle in this.

The APS-EPS Steering Committee on Computational Physics met and endorsed the plan to organize the 1994 meeting in Manno (near Lugano), Switzerland.

R.A. de Groot, Chairman, CPG

\title{
First Application of a New Approach
}

\section{FIRST-PRINCIPLES PARALLEL SIMULATION OF MOLECULAR DISSOCIATION}
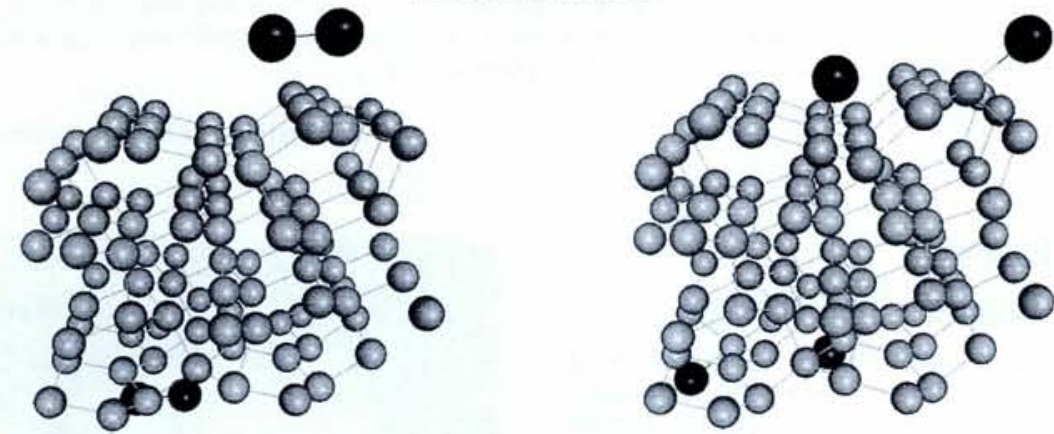

First-principles simulation of chlorine dissociation on the silicon (111) surface. Left and right panels show snapshots of the system as the molecule first approaches the surface, and 0.15 picoseconds later, with chlorine and silicon atoms shown as large and small spheres, respectively.

The last few years have seen a revolution in the computer simulation of condensed matter. Previously, simulations were based on simple models for the interactions between atoms. Increasing computer power is now making it possible to perform fully realistic simulations of matter based on firstprinciples quantum mechanics.

A collaboration between research groups at Cambridge and Keele Universities in the UK reported to the Prague CP ' 92 conference on the first application of first-principles simulation to study molecular dissociation at a solid surface [Stich, I., Payne, M.C.. De Vita, A., and Gillan M.J., CP '92 Poster: First-principles dynamics used to study dissociative chemisorption]. Apart from the new science presented, the work was also technically novel in that the simulations were performed on a massively parallel super- computer - a 64-node Meiko Computing Surface at Edinburgh University. This is the first time that parallel processing has been used for the first-principles simulation of dynamical processes.

The way molecules dissociate when they strike the surface of a solid is important for understanding many different processes, including surface catalysis, corrosion and the operation of gas sensors. There has been a vast amount of experimental work, but many questions remain unanswered. Does the molecule dissociate as it sticks to the surface, or does it stick first and dissociate later? How does the dissociation process depend on the energy and angle of incidence? How do the answers to these questions depend on the molecule and the surface material? First-principles simulation offers for the first time a way of reproducing and analysing what happens when a molecule hits a surface under specified conditions.

The Cambridge-Keele poster presented preliminary results of first-principles simulations of a chlorine molecule striking the (111) surface of silicon. The methods used build on ideas originally proposed in a seminal paper by R. Car and M. Parrinello [Phys. Rev. Lett., 55 (1985) 2471]. For each set of atomic positions as the system evolves in time, Schrödinger's equation for all the valence electrons is solved to determine the electronic ground state. The interaction of these electrons with each atomic core is represented by a "pseudopotential", with electron correlation treated by the local density approximation (LDA). This allows the forces on all the atoms and hence the full time evolution to be calculated. The experience of many research group has demonstrated the accuracy and reliability of pseudopotential-LDA methods.

A number of dynamical simulations were reported, with the chlorine molecule started in different orientations a short distance outside the surface, and projected towards different sites on the surface. The figure illustrates what happens when the molecular axis is initially parallel to the surface. Dissociation occurs spontaneously and very rapidly, and at the same time both chlorine atoms form new chemical bonds with the surface atoms. The indications are that this may be a general feature at the fairly high incident energy of $1 \mathrm{eV}$ used in the present simulations.

The authors stress that the work is still in progress, but what seems clear is that firstprinciples dynamics opens a new way of attacking some unsolved problems concerning molecular processes at surfaces.

M.J. Gillan Keele University, UK 